Revue Revue de l'histoire des religions
de I'histoire des religions
3 | 2018
Varia

\title{
Alex GALLAND, Bouddhisme et christianisme chez Masao
}

Abe

Villeneuve d'Ascq, Presses Universitaires du Septentrion (« Mythes, Imaginaires, Religions »), 2015

Kyong-Kon Kim

\section{OpenEdition}

\section{Journals}

Édition électronique

URL : http://journals.openedition.org/rhr/9265

DOI : $10.4000 /$ rhr.9265

ISSN : 2105-2573

Éditeur

Armand Colin

Édition imprimée

Date de publication : 1 septembre 2018

Pagination : 588-591

ISBN : 978-2-200-93188-9

ISSN : 0035-1423

Référence électronique

Kyong-Kon Kim, "Alex Galland, Bouddhisme et christianisme chez Masao Abe », Revue de l'histoire des religions [En ligne], 3 | 2018, mis en ligne le 01 septembre 2018, consulté le 15 janvier 2021. URL : http://journals.openedition.org/rhr/9265 ; DOI : https://doi.org/10.4000/rhr.9265

Ce document a été généré automatiquement le 15 janvier 2021.

Tous droits réservés 


\section{Alex GALLAND, Bouddhisme et christianisme chez Masao Abe}

Villeneuve d'Ascq, Presses Universitaires du Septentrion (« Mythes, Imaginaires, Religions »), 2015

\section{Kyong-Kon Kim}

\section{RÉFÉRENCE}

Alex Galland, Bouddhisme et christianisme chez Masao Abe, Villeneuve d'Ascq, Presses Universitaires du Septentrion (« Mythes, Imaginaires, Religions »), 2015, 199 p., $24 \mathrm{~cm}$, $24 €$, ISBN 978-2-7574-1140-7.

1 Si, par l'intermédiaire des Stromates de Clément d'Alexandrie (v. 140-v. 220), de l'apparition du manichéisme ( $\mathrm{III}^{\mathrm{e}} \mathrm{s}$.) et de l'Adversus Jovinianum de Jérôme de Stridon (347-420), les contacts entre bouddhisme et christianisme sont à constater dès les premiers siècles de l'ère chrétienne, le dialogue entre ces deux religions plurimillénaires n'a qu'une existence récente. Celui-ci a éclos au siècle dernier et fut jusqu'alors circonscrit par le monologue apologétique et/ou prosélyte qualifiant l'autre soit d'aliénant soit d'idolâtrique. La genèse du dialogue bouddhiste-chrétien fut par ailleurs précédée, d'une part, de l'essor des études philologiques et historiques du bouddhisme introduites par le savant français Eugène Burnouf (1801-1852) et de la collaboration entre les bouddhologues européens et japonais incitée par le gouvernement nippon Meiji (1868-1912) et, d'autre part, des rassemblements des représentants de différentes religions tels que le Parliament of the World's Religions organisé par des chrétiens libéraux à Chicago en 1893. Or, le dialogue intellectuel bouddhiste-chrétien ayant été majoritairement cultivé par des théologiens chrétiens, le cercle des philosophes japonais - l'école dite de Kyōto- qui essaya d'opérer une synthèse entre la pensée bouddhique orientale et la tradition philosophico-religieuse occidentale, tout en explorant une présence analogique de celle-là au sein de celle-ci, et dont fit partie Abe Masao (1915-2006), héritier spirituel de Tanabe Hajime (1885-1962), 
de Nishitani Keiji (1900-1990) et de Hisamatsu Shin'ichi (1889-1981), eux-mêmes influencés (in)directement par Nishida Kitarō (1870-1945), fondateur de cette école, constitue l'un des rares exemples bouddhistes quant à l'engagement pour le dialogue interreligieux et transcontinental.

2 Le présent ouvrage d'Alex Galland $(=\mathrm{AG})$, issu de sa thèse doctorale présentée à l'Université de Lorraine en 2014, examine la plausibilité d'une des théories d'Abe, exposée en 1984 ( « Kenosis and Emptiness ») puis révisée en 1990 ( «enotic God and Dynamic Śūnyatā»). L'essentiel de cette théorie consiste à reconnaître une réalité identique, ou du moins convergente, dans la kenōsis (évidement) christique exposée dans l'Épître de saint Paul aux Philippiens (2.6-11) et dans la śūnyatā (vacuité) universelle, doctrine bouddhique conceptualisée surtout par Nāgārjuna ( $\mathrm{II}^{\mathrm{e}}-\mathrm{III}{ }^{\mathrm{e}} \mathrm{s}$.). Un tel fondement commun offrirait alors aux bouddhistes et aux chrétiens une possible transformation mutuelle et une solidarité face à la montée de l'irreligion dans la société moderne.

Dans la première partie (p. 27-122), après avoir présenté quelques éléments biographiques importants du philosophe japonais (p.13-25), l'auteur procède à l'analyse de la mise en correspondance de la perspective nāgārjunienne de la śūnyatā avec le concept de kenōsis, tout en se référant aux débats des théologiens tels que H. Küng, W. Pannenberg, Th. Altizer ou J. Moltmann. Puis, il déclare irrecevable cette analogie artificielle fondée sur une herméneutique partielle et orientée, en l'occurrence ontologique et non éthique, du terme paulinien « $\dot{\varepsilon} \kappa \varepsilon v \omega \sigma \varepsilon v$ » ainsi que sur une vision partiale, voire hégémonique, de (l'éveil à) la śūnyatā, eu égard à la richesse des doctrines bouddhiques. C'est la raison pour laquelle AG porte son attention sur la théologie naturelle afin de scruter une éventuelle convergence entre la pensée abéienne et la doctrine chrétienne. Toutefois, la cosmologie bouddhique du philosophe niant l'existence d'un Dieu créateur et provident, la théologie naturelle ne pourrait guère prétendre à être un lieu approprié de la rencontre chrétienne avec la réalité ultime abéienne, un «Absolu étranger au régime des causes et des fins » (p. 25).

De ce fait, dans la seconde partie (p.123-181), AG sonde ce lieu de rencontre dans la psychologie spirituelle du pur amour selon, entre autres, le dominicain Alexandre Piny (1639-1709), néanmoins inconnu du philosophe japonais, puisque les conséquences de l'éveil du sujet à la vacuité, c'est-à-dire sa connaissance (skt. vidyā) de son impermanence, de son non-soi (skt. anātman), correspondrait à celles du pur amour envers Dieu, un amour dégagé de tout amour-propre et prêt à renoncer à toute récompense, y compris au salut. Mais, étant donné que cette attitude du pur amour ne relève pas directement de l'orthodoxie chrétienne, il s'avère finalement malaisé de reconnaître une convergence entre la śūnyatā abéienne et le christianisme, si ce n'est une tentative aveugle, en passant sous silence toute divergence, de construire un nouveau paradigme interreligieux.

5 À la lecture de cette monographie, unique en langue française, traitant de la philosophie religieuse d'Abe, il convient de souligner que le comparatisme religieux qui vise à identifier un fonds commun, si passionnant soit-il, se révèle être un exercice fort délicat nécessitant à la fois une précision épistémologique et une rigueur herméneutique. Notamment, si AG, prêtre catholique, a réussi à démontrer, avec justesse et sans aspiration apologétique, la déficience méthodologique de l'analogie abéienne, il aurait été bénéfique d'intégrer, dans l'expertise de la compatibilité « kenōsis-śūnyatā », un exposé historique sur l'évolution du concept « suññatā (pāl.) śūnyatā (skt.) - 空 (chin. kong; jap. kū) - 無 (chin. wu ; jap. mu) - 絶対無 (jap. zettai 
$\mathrm{mu}) »$ au sein du samgha bouddhique ainsi que sur la réception chrétienne de l'idée de "kenōsis", et, dans la seconde partie, une recontextualisation historique de l'émergence de la notion «pur amour ». Si l'auteur a su montrer de façon pertinente l'influence fondamentale de Hisamatsu sur la pensée abéienne, une investigation " généalogique » de la comparaison « kenōsis-śunnyatā », initiée par Nishida (場所的論 理と宗教的世界観, “Logique du lieu et vision religieuse du monde», 1945) et suivie par Nishitani (宗教とは何か, Qu'est-ce que la religion?, 1961), aurait permis de mieux saisir la portée de ce rapprochement dans le contexte japonais où le dialogue bouddhiste-chrétien relevait non seulement d'une rencontre interreligieuse, mais aussi d'une confrontation culturelle et politique. Si AG a pu exposer avec précision la primauté de l'expérience spirituelle (cf.l'« expérience pure» 純粋経験 selon Nishida, 善の研究, Recherches sur le bien, 1911) sur la conceptualisation chez les penseurs du zen et l'inobjectivabilité de la śūnyatā, le terme "l'insaisissable vacuité » (cf.p. 49) semble inadéquate, compte tenu de la perception bouddhique selon laquelle cette réalité serait, contrairement au mystère de Dieu demeurant inaccessible d'après l'intelligence chrétienne, assurément saisissable bien qu'indicible de façon adéquate. Bien que l'honnêteté intellectuelle de l'auteur qui notifie, avec Küng, l'exégèse orientée par rapport aux textes issus d'autres religions, pratiquée tant chez les chrétiens que chez Abe (cf. p. 75s.), soit louable, sa description de l'état post-bodhique souffrant (cf. p. 141; 149) peut être perçue, à son tour, comme une lecture subjective, du point de vue bouddhique qui professe tout le contraire. Quand l'auteur qualifie d'illusoire le cheminement logique d'Abe menant à l'auto-évidement (Selbstentleerung) de l'àtman et du Christ/Dieu, il faut reconnaître, dans le même temps, que la recherche d'un a priori commun des religions entreprise par W.Schmidt (1868-1954), E. Troeltsch (1865-1923), R. Otto (1868-1937), G. van der Leeuw (1890-1950) ou encore M. Eliade (1907-1986), fit souvent l'objet de critique de la part des historiens quant à leur inclination à une reductio ad unum, jugée phénoménologique et idéologique.

6 En outre, bien que ce tractatus philosophicus d'AG ait rendu accessible dans la langue de Molière le débat autour de la théorie d'Abe déroulé dans la langue de Shakespeare, la transcription des termes sanskrits et chinois et la traduction des expressions bouddhiques chinoises auraient mérité d'être plus précises : śūnyatā; kleśa (p. 39); nirvānaa ; saṃsāra; prajñā (p. 52) ; karuṇā (p. 52); jñāna (p. 55); avidyā (p. 59); pāramitā ("perfection » et non «sagesse », p. 59); Fotoroku > (Jiatai) Pudeng lu (p. 64); nirdeśa (p. 67) ; Aller directement à l'esprit, Éveiller sa nature originelle, actualiser sa bouddhéité > Pointer directement l'esprit-cœur de l'homme, Percevoir la nature et devenir le buddha 直指人心 見性成佛 (p. 84); ātman (p.85); Joshû > Zhaozhou (p. 139), etc.

7 Ainsi, cette invitation posthume à une relecture critique, du point de vue chrétien, de la thèse interreligieuse d'Abe met en évidence à la fois l'une des tendances principales du dialogue intellectuel bouddhiste-chrétien, à savoir l'élévation de rencontrecoopération interreligieuse via la reconnaissance des éléments communs, et la complexité de son élaboration, voire son improbabilité, due à l'objectivité lacunaire quant à la compréhension de l'altérité de l'interlocuteur. Sa lecture permettra manifestement d'approfondir la réflexion sur les conditionnements épistémologiques des comparatistes (à la recherche des éléments communs), qui dépendent de leurs propres présupposés socio-culturels et politico-historiques. 


\section{AUTEURS}

\section{KYONG-KON KIM}

Université de Strasbourg. 